\title{
Changes in Conflict, Symptoms, and Well-Being during Psychodynamic and Cognitive-Behavioral Alcohol Inpatient Treatment
}

\author{
Jürgen Hoyer ${ }^{\mathrm{a}}$ Jens Fecht ${ }^{\mathrm{b}}$ Wolf Lauterbach ${ }^{\mathrm{b}}$ Ralf Schneider ${ }^{\mathrm{c}}$ \\ aTechnische Universität, Dresden, bJohann-Wolfgang-Goethe-Universität, Frankfurt am Main, \\ cSalus-Klinik, Friedrichsdorf, Germany
}

\section{Key Words \\ Psychological therapy · Alcohol inpatient treatment • \\ Cognitive conflicts $\cdot$ Symptom severity $\cdot$ Well-being}

\begin{abstract}
Background: According to Grawe's psychological therapy approach, conflict reduction can be expected not only in psychodynamic, but also in cognitive-behavioral therapy (CBT). This was tested in an effectiveness study. Changes in cognitive conflicts, along with those of symptom severity and well-being were analyzed during alcohol inpatient treatment. Methods: Four times during treatment, groups of patients receiving psychodynamic therapy ( $n=45$ patients) or CBT ( $n=49$ patients) were measured and compared. Lauterbach's Online Conflict Test was used to measure conflict. Symptom severity and well-being were measured using questionnaires. $\boldsymbol{R} \boldsymbol{e}$ sults: Results showed significant conflict decrease in both groups with a tendency towards faster reduction under CBT. There was also significant change in symptom severity and well-being in both groups with no difference regarding reduction gradient. Moreover, patients in the psychodynamic treatment group exhibited
\end{abstract}

lower symptom scores at treatment begin which may be a consequence of clinical group assignment. Conclusions: In general, the findings confirmed Grawe's theoretical assumptions. Generalizability to other diagnostic groups and other clinical settings remains to be tested.

Copyright $\odot 2001$ S. Karger AG, Basel

In his recent monograph 'Psychological Therapy', Grawe [1] discussed the concept of psychological conflict within the framework of empirically based treatment in clinical psychology. From this perspective, inner conflict is pertinent to all psychotherapy and not limited to psychodynamic settings. Given the impressive effectiveness of cognitive-behavioral therapies (CBT), the question arises as to what happens to inner conflicts during CBT, since CBT does not address the treatment of conflict. Do conflicts remain untreated and persist? Are they reduced as an epiphenomenon of symptom reduction? Grawe's answer is that CBT affects conflicts indirectly. Problematic attitudes and contradictory motivational tendencies that, for example, constitute approach avoidance conflict may be changed as a consequence of the cognitive restructuring initiated by new behavioral experiences.

\section{KARGER \\ Fax + 41613061234 \\ E-Mail karger@karger.ch \\ www. karger.com

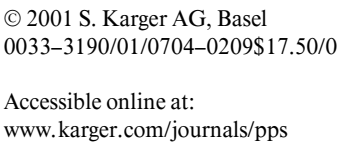

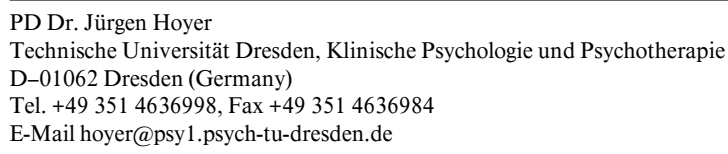

PD Dr. Jürgen Hoyer D-01062 Dresden (Germany)

E-Mail hoyer@psy1.psych-tu-dresden.de 
The case of a social phobic patient who is usually ambivalent about going to parties illustrates this point. This patient may be motivated to find friends and to have contact with other people. At the same time, he/she may feel uncomfortable with the idea of going to a party because he/she expects negative social feedback and embarrassing experiences. CBT challenges these negative assumptions using techniques such as role play or behavior experiments. If this leads to the reduction of negative assumptions, the experiential quality of the situation is less conflict laden, and the accessibility of associated conflicting representations of similar events is reduced.

If this reasoning were correct, we would expect conflicts to be reduced not only in psychodynamic therapy but also in CBT. A study by Renner and Platz [2] tested the hypothesis that standardized cognitive-behavioral group treatment reduces cognitive conflicts as measured by a conflict test based on cognitive dissonance theory [3, 4]. Although conflict reduction was not intended or addressed directly in the therapy sessions, a significant reduction in cognitive conflicts was found in a group of anxiety patients. Within groups of depressed patients and remitted schizophrenic patients, the effect could not be replicated. However, the study did not include a psychodynamic treatment condition and, thus, did not answer whether psychodynamic treatment would result in greater conflict reduction than CBT. Conversely, there are no studies examining changes in conflict using psychodynamic approaches (e.g. as described by Perry et al. $[5,6]$ ) that included a CBT condition.

Therefore, the present investigation aims to compare the extent to which cognitive conflicts are reduced in both CBT and psychodynamic therapy (PT).

Moreover, we will analyze changes in general symptom severity and diverse aspects of psychological well-being, thereby also encompassing factors of positive health [7, 8]. Thus, it is possible to examine how conflict reduction relates to changes of symptom severity and well-being during treatment as well as whether conflicts are closer related to symptom severity or well-being. Relationships between well-being and conflict are of special interest because they have rarely been studied [9].

As previous research has shown, cognitive conflicts are heightened in alcoholics as compared to abstinent alcoholics [10], and these conflicts strongly correlate with symptom severity [11]. Furthermore, the importance of conflicts in the area of substance abuse has also been discussed from a motivational perspective [12]. Therefore, this study was carried out in an alcohol inpatient setting.

\section{Methods}

\section{Overview}

This study was conducted as an effectiveness study in a natural clinical setting. Clinical conditions were representative of alcohol inpatient treatment in Germany. Patients, who were not preselected, were treated by experienced therapists with a regular therapeutic workload. Indicative decisions and treatment were conducted within routine care and not by the investigators. As a consequence, there was no randomized treatment assignment. Assignment to one of the treatment conditions was determined by an experienced diagnostician after a diagnostic review at treatment begin. This review followed clinical requirements and additional criteria such as previous treatments, patient preferences.

Conflict, symptom severity and well-being as well as other variables including self-consciousness [13] were measured four times during treatment. Assessment sessions were conducted in the first or second (t1), fifth or sixth (t2), ninth or tenth (t3) and thirteenth or fourteenth week $(\mathrm{t} 4)$ of treatment.

\section{Treatments}

Treatment consisted of specific and nonspecific interventions. Each patient participated in individual and group therapy sessions as well as in diverse training groups ranging from social skills training to sports or music therapy. Treatment included one individual and one group therapy session per week on average. Therapists were either trained and experienced in CBT or PT. Since every patient participated also in unspecific training groups, psychotherapeutic treatment was not applied exclusively in any of the cases.

\section{Therapists}

Twenty-four therapists collaborated in this study. Sixteen were CBT therapists and 8 were psychodynamic therapists. Therapeutic experience ranged from 2 to 20 years in the CBT group (mean $=7.86$, $\mathrm{SD}=6.34$ ) and from 6 to 20 years in the psychodynamic therapists (mean $=13.8, \mathrm{SD}=5.25)$. Psychodynamic therapists had significantly more experience $[\mathrm{t}(23)=5.71, \mathrm{p}<0.001]$, although all therapists were judged to be experienced.

\section{Selection Criteria}

Selection criteria included a primary diagnosis of alcohol dependency (ICD F10.2) and participation in the clinical routine program lasting 12-18 weeks. Patients with a history of psychosis or neurological deficits were excluded.

\section{Drop-Out and Missing-Value Analysis}

One hundred and thirty patients were included in the study. Of these, 13 patients stopped treatment due to various reasons (relapse, medical condition) while 42 patients produced missing values. To maximize the power for subsequent statistical analyses, when all other assessments were complete, missing data in the data row $(\mathrm{t} 2, \mathrm{t} 3)$ were replaced by the mean of the scores next to the missing ones. This was done in 9 cases for the conflict score and in 10 cases for the symptom and the well-being measures. Of the remaining patients, when conflict was analyzed, 49 were treated using CBT and 45 underwent PT. When symptoms and well-being were analyzed, 47 were in the CBT, and 40 in the PT group. 
drinking alcohol

(I like to have social contacts)

drinking alcohol

Fig. 1. A balanced (harmonious) and an imbalanced (conflict) triad. Relations between concepts ('myself', 'drinking alcohol', 'social contact') are formulated as items in the Online Conflict test (e.g. 'Do you like to have social contacts?' relating the concepts 'myself' and 'social contact'). Answers are analyzed with respect to being contradictory on the basis of triads as shown below.

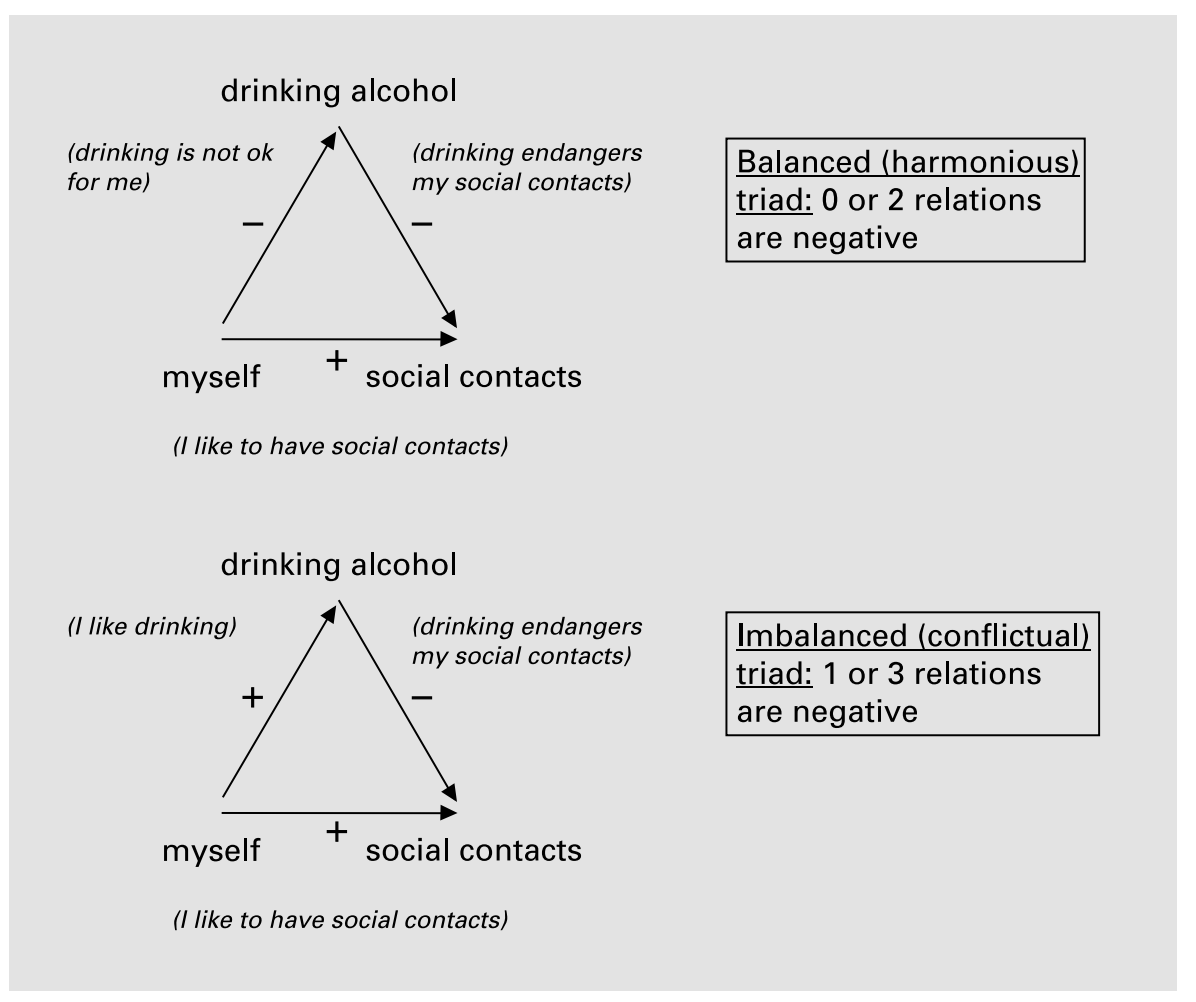

\section{Sample}

Average age of the sample was about 42.5 years. Half of the sample was female, which indicates that the sample is not representative with respect to gender ratio; the proportion of females is higher than found in the population. Patients in the CBT and PT group did not differ with regard to age $[\mathrm{t}(92)=0.08, \mathrm{p}>0.20)$ and gender ratio $\left[\chi^{2}(1, n=94)=1.47, p>0.20\right]$. Approximately one third of the patients had either a low, medium or high educational level. Patients in both groups were equally represented in these three categories $\left[\chi^{2}(2, n=94)=1.05, p>0.20\right]$. Average duration of alcohol problems was 10.0 years $(\mathrm{SD}=7.60)$ in the CBT group and $7.47(4.76)$ years in the PT group $[\mathrm{t}(92)=1.92, \mathrm{p}=0.06]$.

\section{Measures}

Several methods for operationalizing and measuring conflict have been proposed in recent years, many of them based on psychodynamic theory and on rating procedures $[5,6]$. The method used in this study $[3,4]$ facilitates objective assessment of patients' conflicts and their change in therapy. It is based on long-standing empirically validated principles of social psychology, namely cognitive dissonance theory. Conflicts are defined as contradictions between personally relevant topics and their meanings [3, 4]. The computer presents test items, records and stores the patients' answers, and identifies inconsistencies (or contradictions) between patients' attitudes toward individual topics and beliefs about their interaction. The extent of cognitive inconsistency is measured at the level of substructures of triads, i.e. a structure of three cognitive concepts or elements (topics) and the subjective relationships between them. According to the balance theory of Heider [14], a triad is balanced if 0 or
2 relationships are negative. A triad is imbalanced if 1 or 3 relations are negative. This is illustrated in figure 1.

All possible relationships, i.e. attitudes toward the elements, and beliefs about the relationships between the elements, are presented to the participant as items ranging from ' -10 ' to ' +10 '. All possible cognitive triads, each comprised of 3 items, are constructed post hoc by the computer, and their balance is computed. Thus, the test does not contain any direct questions concerning 'conflict'. Only an indicator of the total amount of conflict in the cognitive field, the global conflict index [3], is presented in this research.

This method has proven reliable and valid in its paper-pencil form [3]. Its on-line version, which partially differs with regard to wording, was used for the first time in this study. Therefore, participants were carefully instructed how to answer questions in the PCformat. Based on previous research $[10,11]$, the cognitive field consisted of 7 elements seen as relevant for all participants (myself, partnership, self-confidence, controlled drinking, social contact, active participation in therapy and a satisfying job). Two further elements could be chosen individually by the participants from a list provided by the investigators.

Symptom severity was measured with the German version of the revised Symptom Checklist $[15,16]$. This 90 -item self-rating scale is well established and has proven highly reliable and valid, especially as a measure of general psychopathology [16]. Only its total score, the Global Severity Index (GSI), was used in the present research.

Additionally, to capture different aspects of psychological wellbeing, the scales for measuring habitual mood (HM) and life satisfaction (LS) by Dalbert [17] were used. These scales are modified versions of the Mood Survey [18] and the Satisfaction with Life Scale 
Table 1. Means and standard deviations in conflict, symptom severity, and well-being (habitual mood, life satisfaction) over the course of treatment in both treatment groups

\begin{tabular}{lcccccc}
\hline & & $\mathrm{n}$ & $\mathrm{t} 1$ & $\mathrm{t} 2$ & $\mathrm{t} 3$ & $\mathrm{t}$ (4 \\
\hline Cg & Total group & 94 & $240.54(115.25)$ & $154.04(105.76)$ & $137.14(103.99)$ & $110.65(115.64)$ \\
& PT & 45 & $235.58(106.73)$ & $169.08(111.19)$ & $156.16(106.48)$ & $107.51(115.64)$ \\
& CB & 49 & $245.10(123.48)$ & $140.23(99.66)$ & $119.67(99.55)$ & $113.53(131.78)$ \\
\hline GSI & Total group & 87 & $0.68(0.58)$ & $0.47(0.49)$ & $0.45(0.50)$ & $0.41(0.50)$ \\
& PT & 40 & $0.53(0.40)$ & $0.38(0.36)$ & $0.37(0.39)$ & $0.37(0.45)$ \\
& CBT & 47 & $0.81(0.68)$ & $0.55(0.58)$ & $0.51(0.57)$ & $0.44(0.53)$ \\
\hline HM & Total group & 87 & $3.24(1.12)$ & $3.41(0.94)$ & $3.62(1.00)$ & $3.70(1.03)$ \\
& PT & 40 & $3.33(1.11)$ & $3.48(0.98)$ & $3.66(1.10)$ & $3.70(1.07)$ \\
& CB & 47 & $3.16(1.14)$ & $3.34(0.92)$ & $3.57(0.92)$ & $3.69(1.00)$ \\
\hline LS & Total group & 87 & $3.49(0.96)$ & $3.64(0.88)$ & $3.83(0.93)$ & $3.94(0.91)$ \\
& PT & 40 & $3.51(0.93)$ & $3.65(0.94)$ & $3.79(0.95)$ & $3.88(0.90)$ \\
& CB & 47 & $3.47(1.00)$ & $3.63(0.84)$ & $3.83(0.93)$ & $3.99(0.93)$ \\
\hline
\end{tabular}

$\mathrm{Cg}=$ Global conflict score

[19]. Both scales have proven reliable in large German samples [20]. Moreover, latent structural modeling confirmed the a priori model and indicated construct validity in both scales [17].

\section{Data Analysis}

Data were analyzed by two-way (time, therapeutic orientation) ANOVAs with repeated measures on one factor with global conflict score, or well-being (HM, LS) as dependent variables. For symptom severity, scores did not show homogeneous variances of errors. Also, there were significant differences between the two groups at baseline ( $p=0.040$, Wilcoxon's test) with symptom scores being lower in the PT group. Therefore, multivariate linear regressions were fitted to the data with the second, third and fourth measurements of symptom severity being the dependent variables and the baseline value as an additional covariate. According to the shape of the distribution within the cells, the natural logarithm of the symptom severity scores $\left[\ln \left(x+10^{-5}\right)\right]$ was used instead of the original values. Robust estimates of the standard errors were calculated [21] to determine any deviations from model assumptions of linear regression such as heterogeneous variances of errors. For assessing effect sizes in a noncontrol group design, a formula by McGaw and Glass [22] was applied.

\section{Results}

Descriptive Statistics and Pretreatment Comparisons

Descriptive statistics of both treatment groups in all dependent measures are presented in table 1 .

The GSI of the sample at beginning of treatment is in the lower range to be expected for patients. Fifty-four percent of the sample scored below the cutoff score that
Schauenburg and Strack [23] identified for German psychotherapy samples $(\mathrm{GSI}=0.57)$. This may be due to (1) the clinic not regularly taking patients directly following detoxification and (2) the majority of patients not having previous experience with inpatient treatment and, therefore, being less disordered than more chronic alcohol patients. As noted above, these prescores were not obtained before treatment but rather in the first or second week of treatment, which may have resulted in a beginning 'remoralization' [24] in many of the patients.

With regard to cognitive conflict and well-being, pretest data appear to be very similar to previous data [10, 25]. No differences regarding treatment assignment were related to these variables.

\section{Correlations}

We did not expect differential correlations between treatment groups. Therefore, to reduce complexity, correlational analyses were only conducted for the total sample (table 2).

Both at the beginning and end of treatment, conflict correlated negatively and significantly with psychological well-being. Correlations between conflict and symptom severity (GSI) were positive, but significant only at treatment end. Accordingly, there is a tendency towards a closer relationship of conflict with well-being than with symptom severity. 
Table 2. Correlations between conflict, symptom severity, habitual mood and life satisfaction in 87 alcohol inpatients at beginning and end of therapy

\begin{tabular}{|c|c|c|c|c|}
\hline & $\mathrm{Cg}$ & GSI & $\mathrm{HM}$ & LS \\
\hline \multicolumn{5}{|c|}{ Treatment beginning $(t 1)$} \\
\hline $\mathrm{Cg}$ & - & 0.19 & $-0.29 * *$ & $-0.35^{* *}$ \\
\hline GSI & & - & $-0.51^{* *}$ & $-0.52 * *$ \\
\hline HM & & & & $0.69 * *$ \\
\hline \multicolumn{5}{|c|}{ End of treatment (t4) } \\
\hline $\mathrm{Cg}$ & $(0.24 *)^{1}$ & $0.32 * *$ & $-0.35^{* *}$ & $-0.47 * *$ \\
\hline GSI & & $\left(0.66^{* *}\right)$ & $-0.48^{* *}$ & $-0.51^{* *}$ \\
\hline HM & & & $\left(0.73^{* *}\right)$ & $0.82^{* *}$ \\
\hline LS & & & & $\left(0.62^{* *}\right)$ \\
\hline
\end{tabular}

$\mathrm{Cg}=$ Global conflict score $* \mathrm{p}<0.05, * * \mathrm{p}<0.01$.

1 Pre/post autocorrelation in the diagonal (in parentheses).

Moreover, there is considerable overlap between symptom severity and well-being as indicated by stable medium-sized and significant (negative) correlations between the two variables.

A look at the stability of the variables as indicated by the autocorrelations over time (table 2) showed a relatively high stability of symptom severity and well-being, whereas conflict appears to fluctuate more.

The correlation of $t 1 / t 4$ changes in conflict and $t 1 / t 4$ changes in symptoms was shown to be positive but insignificant $(\mathrm{r}=0.14, \mathrm{p}=0.17)$.

\section{Changes in Conflict, Symptoms and Well-Being}

For conflict, there was a significant main effect of time $[F(3,276)=51.02, p<0.001]$. This was due to significant contrasts between all points in time, except $t 2$ and $t 3$, indicating that therapy is effective in reducing conflicts in general and most so in the first and last phase. Effect size (ES) for pre-post differences (according to McGaw and Glass [22]) was ES $=0.91$. Therapeutic orientation was irrelevant for differences in conflict as a main effect $[\mathrm{F}(1$, $92)=0.46, p=0.50]$. Nevertheless, there was statistical tendency towards an interaction between time and therapeutic orientation $[F(3,276)=2.28, p=0.08]$. Inspection of the group mean scores (fig. 2) reveals that this is due to lower scores of conflict in the CBT condition at $\mathrm{t} 3[\mathrm{~F}(1$, 92) $=9.47, \mathrm{p}<0.01]$.

For symptom severity, there was no evidence for interaction effects of group with time on symptom severity $[F(2,87)=0.96, p=0.388]$ nor between the baseline value

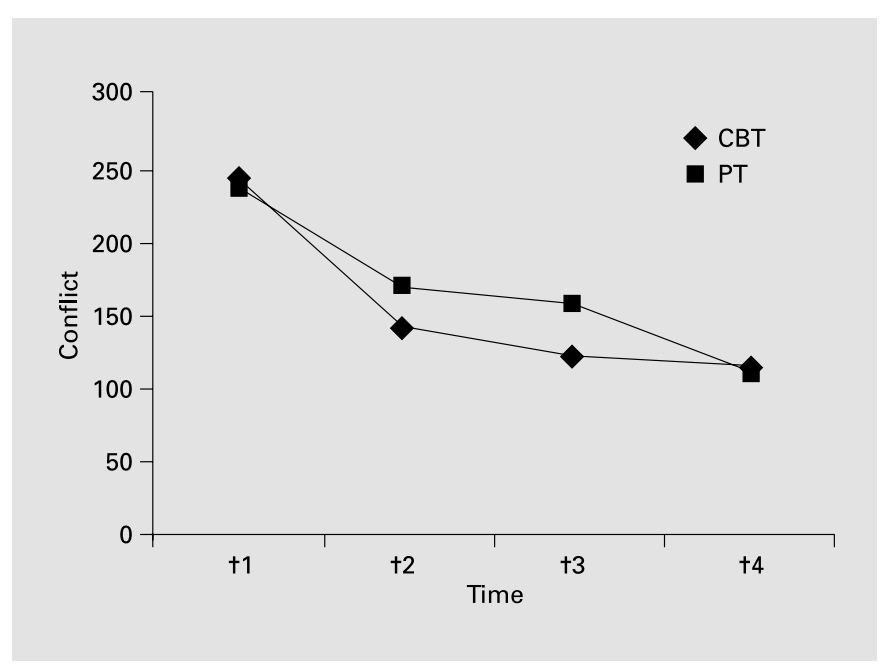

Fig. 2. Conflict reduction in $P T$ and CBT.

and time $[F(2,87)=0.04, p=0.964]$. In the model where the interaction terms were omitted, the baseline values were associated with higher severity scores (regression coefficient $=1.67,95 \% \mathrm{CI}=1.12-2.22, \mathrm{p}=0.000)$, but there was no evidence for the groups differing in symptom severity $\left(r_{c}=0.44,95 \% \mathrm{CI}=-0.25\right.$ to $\left.1.14, \mathrm{p}=0.209\right)$. Over all four assessments there was a time effect $\left(r_{c}=\right.$ $-0.46,95 \% \mathrm{CI}=-0.64$ to $0.28, \mathrm{p}=0.000$ ), and this time effect seemed to be uniform over the different assessments $(E S=0.60)$.

For both well-being measures, a significant main effect of time was found [HM: $F(3,262)=10.24, p<0.001$; LS: $\mathrm{F}(3,262)=8.74, \mathrm{p}<0.001]$ with the pattern of change as already found for conflict, i.e. no changes between $\mathrm{t} 2$ and t3. Effect sizes were at $\mathrm{ES}=0.58(\mathrm{HM})$, and $\mathrm{ES}=0.55$ (LS), respectively. There were no significant group effects or interactions.

\section{Discussion}

In the present investigation, significant reductions of both conflict and symptom severity and significant enhancement of well-being in patients in a natural alcohol inpatient setting were found regardless of whether the therapeutic condition followed a cognitive-behavioral or a psychodynamic rationale. Schulz et al. [27] have recently presented similar data with regard to psychosocial variables in psychosomatic inpatients, although with slightly greater effect sizes (which may be due to the low impair- 
ment of our sample). As there is a tendency towards higher effect sizes in conflict than in symptom severity and well-being, this variable appears to be a sensitive indicator of therapeutic change at least in a 'remoralized' sample showing less impairment. Moreover, conflict correlates with both symptoms and well-being, thus indicating that conflicts are linked to problems in developing or maintaining well-being. These problems may in turn precede relapse [28]. Interestingly, only insignificant correlations between conflict changes and symptom changes were found, indicating that these are not intimately associated epiphenomena of the therapeutic process, but can largely vary independently.

The fact that these findings are based on a naturalistic effectiveness study should be emphasized. This limits the generalizability of results to similar inpatient settings which may be uncommon in other parts of the world, especially in the treatment of alcoholics. On the other hand, the selection bias in such settings may include higher rates of comorbidity and chronicity [29], thus indicating the clinical utility of both CBT and PT in this field. However, due to the naturalistic conditions, the data should be interpreted carefully when looking at the differential effects of therapeutic orientation.

Cognitive conflict was reduced during CBT as well as during PT. CBT patients did not receive manual guided therapy. However, the individualized pattern of therapy interventions they received was based entirely on CBT methods. Therefore, the data confirm Grawe's view that cognitive changes initiated in CBT may lead to an implicit reduction of conflicts. That this tendency towards reduction appears to be even faster under CBT may be explained by the fact that CBT therapists address problematic cognitions of the patient directly and more promptly. However, this is a post hoc explanation and further work as to how CBT therapists actually reduce cognitive conflicts should be performed in the future.

At the end of therapy, PT appears to be equally effective in all measures. This suggests that psychodynamic therapists may have 'worked through' inner conflicts of their patients more thoroughly before they finally reach the goal of their reduction. Such an explanation would be in line with psychodynamic treatment rationale. However, this effect is less clear because this treatment condition can also be seen as a combination of PT (single and group therapy) and CBT (additional training groups).

Generally, an important limitation of our study is the lack of clarity in the proportion of treatment interventions that were actually applied in the two conditions. This should be more explicitly examined in future research.
Accordingly, the interpretation that similar effects in both groups are due to relatively similar treatment cannot be sufficiently ruled out. Furthermore, no pure control group was used. Thus, data cannot be compared with spontaneous fluctuation. However, it seems improbable that untreated alcoholics would have shown a similar course in reduction of conflict and symptoms as well as improvement in well-being.

Moreover, within the possibilities of the present study it remains unclear whether PT would have been even more effective than CBT with regard to conflict reduction if other more psychodynamic methods of operational conflict measurement $[5,6]$ had been used. Therefore, it would be useful to replicate this part of the study using these methods.

Additionally, the unexpected finding of lower symptom severity of $\mathrm{t} 1$ inpatients who were assigned to PT seems of interest. This may indicate that psychodynamic therapists, at least in the clinic under investigation, are seen as more qualified by the diagnostician to treat patients who have personal problems but are less impaired.

Notwithstanding the above-mentioned limitations of the study, our findings, in general, confirmed the theoretical assumptions of Grawe [1]. Clearly, generalizability to other settings, other disorders, and to measures of unconscious conflict remains to be tested and will hopefully be prompted by this article.

\section{Acknowledgement}

This research was supported by grant LA 424/9-1 from the Deutsche Forschungsgemeinschaft (DFG). 


\section{References}

1 Grawe K: Psychologische Therapie. Göttingen, Hogrefe, 1998.

2 Renner W, Platz T: Kognitive und symptombezogene Effekte standardisierter Verhaltenstherapie: Evaluation eines ambulanten Gruppenprogramms. Z Klin Psychol Psychiatr Psychother 1999;47:271-292.

3 Lauterbach W: The measurement of personal conflict. Psychother Res 1996; 6:213-225.

4 Lauterbach W, Newman CF: Computerized intrapersonal conflict assessment in cognitive therapy. Clin Psychol Psychother 1999;6:118 .

5 Perry JC, Augusto F, Cooper SH: Assessing psychodynamic conflicts. I. Reliability of the idiographic conflict formulation method. Psychiatry 1989;52:289-301.

6 Perry JC, Luborsky L, Silberschatz G, Popp C: An examination of three methods of psychodynamic formulation based on the same videotaped interview. Psychiatry 1989;52:302-323.

7 Fava GA, Sonino N: Psychosomatic Medicine: Emerging trends and perspectives. Psychother Psychosom 2000;69:184-197.

8 Ryff CD, Singer BH: Biopsychosocial challenges of the New Millennium. Psychother Psychosom 2000;69:170-177.

9 Emmons RA, King L: Conflict among personal strivings: Immediate and long-term implications for psychological and physical well-being. J Pers Soc Psychol 1988;54:1040-1048.

10 Hoyer J: Kognitive Konflikte bei Alkoholpatienten und abstinenten Alkoholikern. Sucht 1995;41:252-264.
11 Hoyer J, Frank D, Lauterbach W: Intrapsychic conflict and intolerance of ambiguity as predictors of severity of clinical symptoms - A latent variable approach. German J Psychol 1994;18: 351-352.

12 Cox WM, Klinger E: A motivational model of alcohol use. J Abnorm Psychol 1988;97:168180.

13 Hoyer J, Fecht J, Heidenreich T: Selbstaufmerksamkeit und ihre Veränderung bei stationären Alkoholpatienten. Z Klin Psychol Psychiatrie Psychother, in press.

14 Heider F: The Psychology of Interpersonal Relations. New York, Wiley, 1958.

15 Derogatis CR: SCL-90-R: Administration, Scoring and Procedures. Manual I for the R(evisited) Version. Baltimore, Johns Hopkins University School of Medicine, 1977.

16 Franke G: Die Symptom Checkliste von Derogatis - Deutsche Version. Göttingen, Hogrefe, 1995.

17 Dalbert C: Subjektives Wohlbefinden junger Erwachsener: Theoretische und empirische Analysen der Struktur und Stabilität. Z Diff Diagn Psychol 1992;13:207-220.

18 Underwood B, Froming WJ: The mood survey: A personality measure of happy and sad moods. J Pers Assess 1980;44:404-414.

19 Diener E, Larsen XE: The Satisfaction with Life Scale. J Pers Assess 1985;49:71-75.

20 Hoyer J: Dysfunktionale Selbstaufmerksamkeit. Heidelberg, Asanger, 2000

21 Royall RM: Model robust confidence intervals using Maximum Likelihood Estimators. Int Stat Rev 1986;54: 221-226.
22 McGaw B, Glass GV: Choice of metric for effect size in meta-analysis. Am Educ Res J 1980;17:325-337.

23 Schauenburg H, Strack M: Measuring psychotherapeutical change with the Symptom Checklist SCL 90 R. Psychother Psychosom 1999;68: 169-226.

24 Howard KI, Lueger RJ, Maling MS, Martinovitch Z: A phase model of psychotherapy outcome: Causal mediation of change. J Consult Clin Psychol 1993;61:678-685.

25 Heidenreich T: Intrapsychische Konflikte und therapierelevante Variablen: Therapie- und Veränderungsmotivation. Frankfurt am Main Johann Wolfgang Goethe-Universität, 1999.

26 McClelland GH, Judd C: Statistical difficulties of detecting interactions and moderator effects. Psychol Bull 1993;114:376-390.

27 Schulz H, Lotz-Rombaldi W, Koch U, Jürgensen R, Rüddel H: 1-Jahres-Katamnese stationärer psychosomatischer Rehabilitation nach differentieller Zuweisung zu psychoanalytisch oder verhaltenstherapeutisch orientierter Behandlung. Psychother Psychosom Med Psychol 1999;49:114-130.

28 Fava GA: Well-being therapy: Conceptual and technical issues. Psychother Psychosom 1999; 68:171-179.

29 Rief W, Trenkamp S, Auer C, Fichter MM: Cognitive behavior therapy in panic disorder and comorbid major depression. Psychother Psychosom 2000;69:70-78. 